\title{
Predictors of Discrepancy Between Care Recipients With Mild-to-Moderate Dementia and Their Caregivers on Perceptions of the Care Recipients' Quality of Life
}

\author{
Heehyul Moon, PhD', Aloen L. Townsend, PhD², \\ Peggye Dilworth-Anderson, $\mathrm{PhD}^{3}$, and Carol J. Whitlatch, $\mathrm{PhD}^{4}$
}

\begin{abstract}
Purpose: The goal of this study was to explore predictors of discrepancy between reports of caregivers (CGs) and care recipients (CRs) with mild-to-moderate dementia about CRs' quality of life (QOL). Design and Methods: This study was a secondary analysis of cross-sectional data drawn from a study of 200 care dyads of CRs with mild-to-moderate dementia and their primary family CGs. Paired $t$ test, ordinary least squares multiple regression, and binary logistic regression were used for the analyses. Results: Caregivers rated CRs' QOL significantly lower (worse) than CRs did. Ordinary least square regression results showed that greater incongruence in perceptions of CRs' decision-making involvement (DMI) and higher level of CR impairment in activities of daily living were significantly related to higher absolute discrepancy between CG and CR about CRs' QOL. In the logistic models, when the dyad had more DMI incongruence, or CG reported higher relationship strain, the CG was more likely to report a lower CR QOL than CR reported. Implications: Practitioners should consider incorporating CRs' perspective when planning care instead of solely depending on CGs' perspective. Also, practitioners should pay attention to any gap between perceptions of CGs and CRs, particularly with regard to CRs' QOL.
\end{abstract}

\section{Keywords}

mild-to-moderate, dementia, quality of life, discrepancy

\section{Introduction}

Nearly five and a half million Americans aged 65 years and older have Alzheimer's disease, the sixth-leading cause of death in the United States, and this number is expected to increase to 9 million by $2030 .{ }^{1}$ Reflecting this demographic trend, gerontological studies have increasingly focused on dementia., ${ }^{2,3}$

Recent studies have suggested that, despite a noticeable decline in cognitive function, individuals with mild-tomoderate dementia are capable of expressing their thoughts and preferences ${ }^{4,5}$ and want to express their needs and views about the caregiving process. ${ }^{6-8}$ However, care recipients (CRs) are frequently excluded from their own care processes because of popular stereotypes about their cognitive function, resulting in CR perceptions (eg, quality of life [QOL]) being frequently ignored by caregivers (CGs) and health-care providers. ${ }^{4}$ However, these feelings of exclusion by CRs may be eliminated or reduced if CRs were cared for from personcentered approach, which involves treating people as individuals, looking at the world from the perspective of the person with dementia, providing a positive social environment to facilitate well-being, and placing the person with dementia at the center of the care dynamic rather than to place emphasis on the condition a person may have. ${ }^{9}$

Quality of life has been defined and measured in many different ways but typically includes psychological, physical, and social well-being. ${ }^{10}$ Rubinstein and Lawton, ${ }^{11}$ based on a person-centered approach, was the first study to emphasize the importance of getting information directly from persons with

\footnotetext{
' Raymond A. Kent School of Social Work, University of Louisville, Louisville, KY, USA

${ }^{2}$ Mandel School of Applied Social Sciences, Case Western Reserve University, Cleveland, $\mathrm{OH}$, USA

${ }^{3}$ Gillings School of Global Public Health, The University of North CarolinaChapel Hill, Chapel Hill, NC, USA

${ }^{4}$ Benjamin Rose Institute on Aging/Center for Research and Education, Cleveland, $\mathrm{OH}, \mathrm{USA}$
}

\section{Corresponding Author:}

Heehyul Moon, PhD, Raymond A. Kent School of Social Work, University of Louisville, Oppenheimer Hall 210, Louisville, KY 40292, USA.

Email: heehyul.moon@louisville.edu

This article was accepted under the editorship of the former Editor-in-Chief, Carol F. Lippa. 
dementia as well as others or observation and of investigating QOL of CRs with dementia. While there is no consensus on how to measure QOL of people with dementia, Rubinstein and Lawton noted that both subjective and objective components should be used. Domains of time use quality, social interaction, and affect states and direct observation in domains of behavior, affect display, and environmental quality should be assessed. ${ }^{11}$ Despite Rubinstein and Lawton's recommendation, dementia research on CR's QOL has typically used CG proxy reports. ${ }^{12}$ One noteworthy exception is research by Logsdon and colleagues ${ }^{10,13}$ who report evidence for the reliability and validity of their QOL measure (covering behavioral competence, psychological status, physical functioning, and interpersonal environment) for both CRs with cognitive impairment and their CGs. Only a few studies have investigated whether there is any discrepancy between CG and CRs with dementia in their perceptions of the CR's QOL. Nine studies have shown that family CGs rate CRs with mild-to-moderate dementia as having significantly worse QOL than CRs themselves do. ${ }^{14-22}$

Understanding what contributes to CG-CR discrepancy concerning the QOL of a $\mathrm{CR}$ with mild-to-moderate dementia is critical for alleviating potential stress caused by the discrepancy, properly assessing the CR's QOL, and planning interventions to support family member CGs and CRs. Discrepancy between dyadic partners in understanding the same situation and disagreement on resolving associated problems can cause other stressors to develop, such as negative relationship quality, a person's inability to cope with stressful events, and misunderstandings and conflict within the family. ${ }^{23,24}$

As suggested by a previous study ${ }^{24}$ from a family systems perspective, there are factors that might contribute to discrepancies, including structural factors, such as needs and proximity, and psychological factors (eg, relationship importance). In addition, dementia caregiving research has provided evidence that background factors such as the CG's race and culture, ${ }^{25}$ the CG's income adequacy, ${ }^{26}$ and the CG's kin relationship with $\mathrm{CR}^{27}$ might affect differentially the caregiving experience of CGs and CRs. However, limited attention has been given to the effects of potential predictors on discrepant perceptions of the CRs' QOL by CGs and dementia CRs. For example, only 3 of the 9 studies cited previously identified the following predictors of discrepancies between CGs and CRs about the CR's QOL: CG reports of CR's disturbing behaviors, CG's perceived distress, CG's QOL, and CG-CR relationship quality $^{22}$; CR's cognitive and physical status ${ }^{18}$; and the burden reported by CGs and depressive symptoms reported by CRs. ${ }^{16}$

However, none of those 3 studies used a conceptual model to guide the selection of potential predictors of CG-CR discrepancy. The Stress Process Model $\left(\mathrm{SPM}^{28}\right)$, which has been widely used in stress coping research for CGs and individuals with dementia, was adapted to conceptualize the model for this study. The SPM includes multiple domains: primary stressors that "emerge solely from the experience of living with dementia" and "the potential distress that results," such as functional status ${ }^{29(\mathrm{p} 297)}$; secondary tensions that are produced by the primary stressors and lie "outside the caregiving situation" ${ }^{30(\mathrm{p} 588)}$; mediators or moderators, such as social support, that influence the associations between the stressors and caregiving outcomes; background characteristics, including sociodemographic attributes, that may influence other domains; and outcomes - for example, well-being - that are influenced by complex interaction among the domains in the SPM to illustrate how stressful caregiving experiences directly and indirectly affect outcomes. ${ }^{29-31}$

Researchers have used the various components of the SPM to test the associations between the components and other distress responses to stressful life circumstances. ${ }^{32}$ However, the SPM as originally formulated is limited in its ability to explain how dyadic stressors between CG and CR might influence the dementia caregiving process because Pearlin's model focuses on how individual/intrapersonal factors may be related to individual-level outcomes. Given the need for dyadic research in caregiving, all possible factors that can affect the dyadic relationship - including individual-level CG and CR stressors and well-being, interindividual or dyadic stressors between CGs and CRs, and both parties' well-being - need to be incorporated to understand more fully the caregiving experience. The present study conceptualized the subjective primary stressors at the interindividual level as incongruence (discrepancy) between CGs' and CRs' reports of the CRs' decision-making involvement. For this study, 3 primary domains in the SPM - background characteristics, primary stressors, and secondary strain - were used to explain the variability in CG-CR belief discrepancy. Relevant components of the model included various CG background characteristics (race, kin relationship with $\mathrm{CR}$, and income adequacy), the primary stressors (functional impairment, DMI incongruence, CR's depressive symptomatology), and secondary strain (CG-CR relationship strain reported by the $\mathrm{CG}$ ).

Given the gaps in our knowledge about CG-CR discrepancies and potential predictors of QOL discrepancies in CG-CR dyads during mild-to-moderate dementia, the aims of the present study were to explore (1) the potential discrepancy between CRs' and CGs' perceptions about CRs' QOL and potential predictors of (2) absolute discrepancy and (3) direction of discrepancy (when CG reports lower CR's QOL than CR). This study adopted the definition of QOL (behavioral competence, psychological status, physical functioning, and interpersonal environment) proposed by Logsdon and colleagues. ${ }^{10,13}$

\section{Methods}

\section{Sample}

Inclusion criteria required that CRs (1) have a confirmed diagnosis of a dementia condition or a Mini-Mental State Examination (MMSE) score between 13 and 26, (2) live at home at the time of their first interview, and (3) have a family CG. Thus, CRs with severe cognitive impairment (MMSE $=0-13$ ), no cognitive impairment (MMSE 26-30), no family CG, or who were hospitalized or institutionalized were excluded. Data were collected through separate in-person interviews with the eligible CGs and CRs. After excluding cases with missing values on any study measures $(n=68)$, the sample was comprised of 200 
dyads, each consisting of a community-dwelling CR with mildto-moderate dementia and her or his primary family CG (for details please see Feinberg and Whitlatch ${ }^{33}$ ).

\section{Measures}

Outcome. The 13-item Quality of Life in Alzheimer's Disease Scale developed by Logsdon et $\mathrm{al}^{10,19}$ was used to assess CR QOL. Logsdon and colleagues ${ }^{10}$ reported evidence that this measure is reliable and valid for both CG and CR. In the present study, both the CRs and the CGs were asked to rate the CR's QOL (CR's $\alpha=.85$; CG's report about CR's QOL $\alpha=$ .83). Using response categories of $1=$ poor to $4=$ excellent, the 13 items assess general QOL: physical health, psychological health, interpersonal relationships, and social environment. Discrepancy between CG and CR responses regarding CR's QOL was operationalized in 2 different ways: an absolute discrepancy measure and a categorical "direction of discrepancy" measure. The absolute discrepancy scores are useful to identify the magnitude of discrepancy - in other words, a higher score indicated a greater discrepancy between $\mathrm{CG}$ and $\mathrm{CR}$ about CR's QOL. However, the absolute discrepancy scores do not provide information about the direction of the discrepancy. Thus, the categorical direction of discrepancy was created to preserve direction of the discrepancy. If the CR's answer was higher than the CG's answer $(\mathrm{CG}<\mathrm{CR})$, the dyad was coded 1 . If the CG's answer was higher than the CR's answer (CG > $\mathrm{CR}$ ), the dyad was coded as 0 . Due to the small number of dyads where they both had the same score (ie, no discrepancy, $C G=C R, n=11$ ), these dyads were combined with the dyads with $\mathrm{CG}$ ratings higher than $\mathrm{CR}$ and were coded as $0(\mathrm{CG} \geq \mathrm{CR})$.

\section{Primary Stressors}

Functional impairment. Functional impairment was measured by CGs' reports of CRs' functioning on the Katz Index of Activities of Daily Living $\left(\mathrm{ADLs}^{34}\right)$ and the Instrumental ADLs $\left(\right.$ IADLs $^{35}$ ). Caregivers reported how often CRs had problems, from $0=$ never to $4=$ daily or more often, with 4 ADLs (dressing, bathing/showering, toileting, and feeding self) and 4 IADLs (preparing meals, medication, household chores, and managing money). Activities of daily living and IADLs have been widely used to assess functional impairment of individuals. As previous studies suggested, ${ }^{36,37}$ combining ADL and IADL items can improve validity of each scale. In addition, in this study, ADLs and IADLs were strongly correlated $(r=$ .63), so they were combined into a single functional impairment scale, with higher scores indicating more impairment $(\alpha=.79)$.

Decision-making incongruence. The 15-item Decision-Making Involvement Scale $\left(\mathrm{DMI}^{38}\right)$ was used to assess CGs' and CRs' thoughts about CRs' involvement in decisions about daily care, such as when to get up, using a scale of $0=$ not at all involved to $3=$ very involved. Incongruence between CGs and CRs was operationalized as an absolute difference score.

Care recipients depressive symptomatology. Care Recipients' reports of their depressive symptomatology was measured by the 20-item Center for Epidemiologic Studies-Depression scale $\left(\mathrm{CES}-\mathrm{D}^{39}\right)$; most items were coded $0=$ rarely or none of the time to $3=$ most of time, but positively worded items were reverse coded. Higher scores indicate more depressive symptomatology $(\alpha=.57)$

\section{Secondary Strain}

Caregiver-Care recipient relationship strain. The 4-item subscale drawn from the Dyadic Relationship Strain scale ${ }^{40}$ assessed the CGs' perspective of relationship strain associated with providing care $(0=$ strongly disagree to $3=$ strongly agree). Higher scores indicate more perceived strain $(\alpha=.793)$.

Background factors. These included CG race, CG kin relationship with $\mathrm{CR}$, and $\mathrm{CG}$ income adequacy. Caregiver race was coded as $0=$ nonwhite (African American $=23 \%$, Hispanic $=$ $9 \%$, Asian $=2 \%$, and others $=2 \%$ ) and $1=$ white. Kin relationship with the CR originally had 9 response options but was recoded into 2 categories (spouse $=0$ and nonspouse $=1$ ) because there were too few cases in the son, daughter, sonin-law, daughter-in-law, sibling, grandparents, or other category. Caregivers' income adequacy asked, "Do you have enough money for basic needs?" and responses were coded from $0=$ strongly disagree to $3=$ strongly agree.

\section{Analysis Strategy}

Descriptive statistics were explored for CGs' and CRs' demographic characteristics. Pearson's correlation assessed the magnitude and direction of bivariate relationships between all study variables, as well as potential multicollinearity between predictors, which was indicated by $r \geq .70 .^{41(\mathrm{p} 60)}$ In order to understand the extent to which CGs and CRs have different perceptions about CRs' QOL (aim 1), a 2-tailed paired $t$ test was used. ${ }^{42}$ Ordinary Least Squares multiple regression analysis with simultaneous entry of predictors was used to investigate predictors of QOL absolute discrepancy (aim 2). A binary logistic regression was also conducted to explore predictors of CGs reporting worse CR QOL than CRs did (aim 3). Goodnessof-fit tests of the model included the $-2 \log$ likelihood, the Hosmer and Lemeshow goodness-of-fit test, and the model $\chi^{2}$. Wald $\chi^{2}$ was used in logistic regression to test statistical significance of the effects of predictors. For all intervallevel study variables, skewness and kurtosis were within normal range, with skewness $<2.0$ and kurtosis $<7.0^{43}$; maximum skewness was .952; and kurtosis was .630 for DMI incongruence. 
Table I. Description of Caregivers $(\mathrm{N}=200)$ and Care Recipients $(\mathrm{N}=200)^{\mathrm{a}}$

\begin{tabular}{lccc}
\hline Variables & $\begin{array}{c}\text { Mean (SD) } \\
\text { or } \%\end{array}$ & $\begin{array}{c}\text { Potential } \\
\text { Range }\end{array}$ & $\begin{array}{c}\text { Observed } \\
\text { Range }\end{array}$ \\
\hline CG age & $64.05(12.67)$ & & $35-9$ I \\
CR age & $75.88(9.06)$ & & $5 \mathrm{I}-97$ \\
CG gender (female) & $79.00 \%$ & & \\
CR gender (female) & $49.50 \%$ & & \\
CG/CR race & & & \\
white/caucasian & $64.00 \%$ & & \\
CG-CR relationship & $56.50 \%$ & & \\
Spouse & $2.22(0.53)$ & $0-3$ & $0-8$ \\
CG income adequacy & $3.46(2.05)$ & $0-32$ & $0-32$ \\
Functional impairments & $9.41(4.94)$ & $0-45$ & $23-5 \mathrm{I}$ \\
DMI incongruence & $37.27(6.08)$ & $0-60$ & $0-12$ \\
CR depressive & & & \\
$\quad$ symptomatology & $4.09(7.24)$ & $0-12$ & \\
CG-CR relationship & & & \\
$\quad$ strain & &
\end{tabular}

Abbreviations: CG, caregiver; $C R$, care recipient; DMI, decision-making involvement; SD, standard deviation.

aln order to increase statistical power, CG age, CG gender, CR age, and CR gender were only used descriptively, not in the regression analysis

${ }^{\mathrm{b}}$ Caregivers and care recipients were the same race.

\section{Results}

\section{Study Sample}

Table 1 presents descriptive information on background characteristics of the CGs and CRs as well as descriptive information about DMI incongruence and relationship strain. For instance, on average, CGs were in their early $60 \mathrm{~s}$ and CRs in their middle 70s; however, the sample contained a wide range of ages, particularly among the CGs.

Bivariate correlations (Table 2) indicated that greater QOL discrepancy was only significantly associated with greater DMI incongruence and more CR functional impairment. The bivariate correlations indicated no multicollinearity and the highest correlation was between CG race and CG relationship with CR, that is, white CGs being more likely to be spouses $(r=-.435)$.

\section{Quality of Life Discrepancy}

Table 3 presents descriptive information on CGs' and CRs' reports about CR's QOL (aim 1). There was an absolute discrepancy in CR's QOL, on average about a 6-point difference, and the discrepancy was statistically significant, $t(199)=$ $-7.852, P<.001$. Caregivers reported significantly lower QOL for CRs than the CRs did.

When the direction of discrepancy between CGs and CRs was examined, in $36 \%$ of the dyads $(n=72)$, either CGs reported higher (better) CR QOL scores than did the CRs (n $=61)$ or the dyads $(\mathrm{n}=11)$ were congruent in their perceptions of the CR's QOL. In less than two-thirds of the dyads ( $\mathrm{n}=$ 128), CGs reported lower (worse) CR QOL than did the CRs.

\section{Regression Analyses}

The second and third aim concerned the associations between the primary stressors (functional impairment, DMI incongruence, and CRs' depressive symptomatology), the secondary strain (CG-CR relationship strain), and the outcome (discrepancy in CRs' QOL), controlling for background factors. As shown in Table 4, the OLS regression model explained $13 \%$ of the variance in absolute discrepancy, a medium effect size. ${ }^{44}$ Results showed that for every 1-point higher in CR functional impairment, absolute discrepancy in CR's QOL was .627 points higher. For every 1-point higher in DMI incongruence, absolute discrepancy in CRs' QOL was .101 points higher. These were the only 2 statistically significant predictors. No background factors were significantly associated with QOL absolute discrepancy.

Table 5 presents the logistic regression results. The $-2 \log$ likelihood was $187.17(d f=7, P<.001)$, meaning the model with the 7 predictors included is a better fit than the null model (the intercept-only model). The Hosmer and Lemeshow goodness-of-fit test was not significant, representing a good model fit and indicating that the observed and expected values are similar for this model. The classification table (not shown) indicated that the model can correctly predict the dyads with CGs reporting worse QOL for CR than CRs did $72.9 \%$ of the time. Wald statistics indicated that 2 variables (DMI incongruence, CG-CR relationship strain) significantly predicted the CG's lower report (than CR's report) on CR's QOL. The odds ratio for DMI incongruence indicates that for each 1-point higher in DMI incongruence, the odds of CG reporting lower CR QOL were 1.09 times higher. The odds ratio for CG-CR relationship strain indicates that for each 1-point higher in CGCR relationship strain, the odds of CG reporting lower CR QOL were 1.18 times higher.

\section{Discussion}

The results reveal that CGs and CRs in this study have significantly different perceptions about CRs' QOL. In accordance with prior studies, ${ }^{22}$ on average CGs reported lower QOL for CRs than CRs did. One possible explanation for this discrepancy may be CGs' own negative feelings about dementia. ${ }^{22}$ Caregivers may internalize the experience of dementia that has caused the CR's deterioration in memory, thinking, behavior, and the ability to perform daily activities. Moreover, CGs' perceived burden of caregiving ${ }^{10}$ may influence their ratings of CRs' QOL. Possibly, CGs and CRs may not have enough communication about CRs' QOL or CGs may assume they already understand CR's QOL because of the amount of time they spend with CRs. ${ }^{45}$ The results from this study suggest that to offset any potential bias particularly in CGs' reports of CR's QOL, it is important to consider the CRs' perceptions of their own QOL.

Our finding that higher functional impairment is significantly associated with more discrepancy in CRs' QOL contradicts findings reported by Sands et $\mathrm{al}^{16}$ who did not find any 
Table 2. Correlations Between Study Variables ( $N=200$ Dyads).

\begin{tabular}{lccccccc}
\hline Variables & $\mathrm{I}$ & 2 & 3 & 4 & 5 & 6 & 7 \\
\hline CG race (I = white) & - & & & & & & \\
CG relationship to CR (I = nonspouse) & $-.435^{\mathrm{a}}$ & - & & & & & \\
CG income adequacy & $.209^{\mathrm{a}}$ & $-.192^{\mathrm{a}}$ & - & & & & \\
CR functional impairment & -.035 & $.192^{\mathrm{a}}$ & $-.186^{\mathrm{a}}$ & - & & & \\
DMI incongruence & -.037 & -.021 & -.028 & $.368^{\mathrm{a}}$ & - & & - \\
CR depressive symptomatology & -.020 & $.152^{\mathrm{b}}$ & -.096 & .075 & -.109 & - & \\
CG-CR relationship strain & -.006 & -.073 & -.097 & $.155^{\mathrm{b}}$ & .134 & $.143^{\mathrm{b}}$ & -.005 \\
QOL absolute discrepancy & .016 & .000 & -.103 & $.323^{\mathrm{a}}$ & $.253^{\mathrm{a}}$ & .113 & -.005 \\
\hline
\end{tabular}

Abbreviations: CG, caregiver; CR, care recipient; DMI, decision-making involvement; $Q O L$, quality of life.

${ }^{\mathrm{a} P} \leq .0 \mathrm{l}$.

${ }^{\mathrm{b}} \mathrm{P} \leq .05$.

Table 3. Descriptive Statistics of CGs' and CRs' Report About CR's Quality of Life ( $N=200$ dyads).

\begin{tabular}{|c|c|c|c|}
\hline Measure & M or $\%$ & SD or $n$ & Potential Range \\
\hline \multicolumn{4}{|l|}{ Quality of life } \\
\hline \multicolumn{4}{|l|}{ Individual scores } \\
\hline $\mathrm{CG}^{\mathrm{a}}$ & 33.39 & 6.44 & $13-52$ \\
\hline $\mathrm{CR}^{\mathrm{a}}$ & 37.60 & 6.08 & $13-52$ \\
\hline Absolute discrepancy ${ }^{b}$ & 6.23 & 6.96 & $0-39$ \\
\hline \multicolumn{4}{|l|}{ Direction of discrepancy } \\
\hline$C G>C R^{b}$ & $30.5 \%$ & $\mathrm{n}=6 \mathrm{I}$ & \\
\hline$C G<C R^{b}$ & $64.9 \%$ & $\mathrm{n}=128$ & \\
\hline$C G=C R^{b}$ & $5.5 \%$ & $\mathrm{n}=\mathrm{II}$ & \\
\hline
\end{tabular}

Abbreviations: CG, caregiver; $C R$, care recipient; $M$, mean; SD, standard deviation; QOL, quality of life.

${ }^{\mathrm{a}} 400$ individuals.

b200 dyads.

Table 4. Results of Simultaneous OLS Multiple Regression Predicting Absolute Discrepancy in CG and CR Reports of CR's Quality of Life ( $N=200$ Dyads).

\begin{tabular}{lcc}
\hline Predictors & $b$ & $\mathrm{SE}_{b}$ \\
\hline CG race (I = white $)$ & .262 & .780 \\
CG relationship to CR (I = nonspouse $)$ & -.414 & .777 \\
CG income adequacy & -.570 & .660 \\
CR functional impairment & $.627^{\mathrm{a}}$ & .183 \\
DMI incongruence & $.101^{\mathrm{b}}$ & .050 \\
CR depressive symptomatology & -.010 & .047 \\
CG-CR relationship strain & .082 & .125 \\
$R^{2}$ & .133 & \\
$F(199)$ & $4.215^{\mathrm{a}}$ & \\
\hline
\end{tabular}

Abbreviations: CG, caregiver; CR, care recipient; DMI, decision-making involvement; OLS, ordinary least square.

${ }^{\mathrm{a}} \mathrm{P} \leq .001$.

${ }^{\mathrm{b}} \mathrm{P} \leq .05$.

significant relationship between $\mathrm{CR}$ functional impairment (combined ADL and IADL) and CG-CR discrepancy in CR's QOL. Family CGs may feel less confidence in their CRs' cognitive and physical functions during mild-to-moderate dementia than the $\mathrm{CR}^{42}$ and may have taken $\mathrm{CRs}$ ' cognitive impairment into consideration when they reported about CRs' QOL. It may also be that CGs are anticipating that the CR's dementia will get worse over time, thus requiring changes in existing medical, financial, or service arrangements. ${ }^{46}$ Previous studies ${ }^{42,47}$ have suggested that CGs tend to report higher level of functional impairment than CRs report. Possibly, because the current study used CG's rating on CR's functional impairment, the relationship might be different if CR's rating on his or her own functional impairment is included.

Incongruence on DMI is predictive of QOL discrepancy using the absolute discrepancy and the direction of discrepancy in CR's QOL. This unique finding may reflect that greater incongruence in one domain may affect perceptions of another domain. Decision-making involvement incongruence may also be an important predictor of QOL. However, the current study did not include the direction of discrepancy in CR's DMI. Future research should examine the relationship between direction of discrepancy in CR's DMI and in CR's QOL.

When the direction of discrepancy in QOL was considered, a greater degree of incongruence on DMI between CG and CR was associated with higher odds of the CG reporting a lower CR QOL than did the CR. Previous research has repeatedly found that CGs are likely to report CRs' disabilities as being more significant than CRs. ${ }^{42,47}$ It is possible that, in assessing the CRs' QOL and involvement in decision-making, CGs may incorporate anticipated problems, such as gradual memory loss, disorientation, personality changes, loss of language skills, and behavioral disturbances, along with stereotypes about CRs with dementia (eg, inaccuracy in memory, incompetence, and intellectual deficits) ${ }^{48}$ resulting in less appreciation for the CR's involvement on decision-making and a lower rating of CRs' QOL.

This study found that higher CG-CR relationship strain reported by the CG was related to higher odds of the CG's lower report on CR's QOL, when the direction of discrepancy in CR's QOL was considered as the outcome. The stress from emotional, physical, and social demands and the conflicts in caregiving often challenge the relationship quality between CRs and their primary CGs. ${ }^{42}$ Possibly, CGs may project their 
Table 5. Logistic Regression Results for the CG Reporting Lower CR's QOL Than CR ( $N=200$ Dyads).

\begin{tabular}{lcccccc}
\hline Predictors & $\beta$ & SE $\beta$ & Wald's $\chi^{2}$ & df & $P$ & Odds Ratio \\
\hline CG race (I = white) & .74 & .42 & 3.06 & I & .08 & 2.10 \\
CG relationship to CR (I = nonspouse) & .07 & .42 & 0.03 & $\mathrm{I}$ & .87 & 1.07 \\
CG income adequacy & .19 & .37 & 0.26 & $\mathrm{I}$ & .61 & .21 \\
CR functional impairment & .07 & .10 & 0.53 & $\mathrm{I}$ & .47 & 1.08 \\
DMI incongruence & .08 & .03 & 8.53 & $\mathrm{I}$ & .003 & 1.09 \\
CR depressive symptomatology & -.03 & .03 & 1.76 & $\mathrm{I}$ & .18 & .97 \\
CG-CR relationship strain & .16 & .07 & 5.44 & $\mathrm{I}$ & .02 & 1.18 \\
Test & $\chi^{2}$ & $\mathrm{df}$ & $P$ & & & \\
Overall model evaluation & 31.152 & 7 & .000 & & & \\
$\quad$ Block & 31.152 & 7 & .000 & & & \\
Model & 11.321 & 8 & .184 & & & \\
$\quad$ Hosmer and Lemeshow & 187.17 & & & & & \\
$\quad$-2 Log likelihood & & & & & \\
\hline
\end{tabular}

Abbreviations: CG, caregiver; CR, care recipient; DMI, decision-making involvement; $\mathrm{QOL}$, quality of life; SE, standard error.

negative feelings stemming from the caregiving onto their ratings of CRs' QOL.

Unlike the prior study by Sands et al, ${ }^{16}$ CR's depressive symptomatology was not a significant predictor of QOL discrepancy. Sands et al used different measures from the current study: the Geriatric Depression Scale ${ }^{49}$ and Brod et al's ${ }^{50}$ Quality of Life questionnaire. Using different measures may impact the results. However, similar to previous studies, ${ }^{16,18,22}$ none of the background characteristics were significant in the present study. The effects of CG-CR relationship strain and DMI incongruence on QOL discrepancy were stronger than the effects of background characteristics (ie, race, income adequacy, kin relationship). Possibly, as Sands and colleagues suggested, primary stressors and secondary strain may predict QOL discrepancy better than background characteristics do. A larger and more diverse sample (on race, kin relationship, and socioeconomic status) may be needed to detect the effects, if any, of background characteristics.

Our findings support the utility of Pearlin et al's $\mathrm{SPM}^{30}$ for investigating potential predictors of CG-CR QOL discrepancy. The primary stressors (functional impairment, DMI incongruence) and secondary strain (CG-CR relationship strain) were significant predictors of $\mathrm{CG}-\mathrm{CR}$ discrepancy. Future studies should look at the effect of CG-CR QOL discrepancy as an additional source of stress on CG and CR well-being.

Our results may prove useful to practitioners who should consider person-centered care ${ }^{51}$ to improve CR's QOL by incorporating CRs' perspective when planning care. Practitioners might also actively consider the importance of engaging dyads in structured discussions that enhance mutual understandings of the similarities and differences between the perceptions of CGs and CRs with regard to CRs' care and QOL - for example, practitioners might provide opportunities to CGs and CRs to express their thoughts about the CRs' care experience and to communicate any differences in perceptions. ${ }^{52}$ This may help service providers understand CGs' and CRs' experiences and implement person-centered care plans both for CGs and for CRs. Finally, practitioners might assess relationship strain and provide opportunities to relieve the strain and promote healthy, supportive relationships between CGs and CRs.

Several weaknesses of the current study include the use of a limited number of predictors and covariates; the inability to distinguish specific types of nonspouse CGs; a crosssectional design that prevents drawing any causal conclusions; and the relatively small sample size. Future research should replicate and extend this study's findings. For example, future studies should examine whether other stressors drawn from the SPM or CRs' reports about their own functional impairment may influence the discrepancy of ratings of CRs' QOL.

Despite the limitations, this study extends current knowledge in several ways. This study provides additional evidence that CRs with mild-to-moderate dementia and their CGs have significantly different perceptions about CRs' QOL. It also highlights the advantages of including the absolute discrepancy and the direction of discrepancy as alternative ways of investigating discrepancies. This study demonstrates the value of applying a theoretical model to investigate predictors of $\mathrm{CG}^{-}$ CR discrepancy in CRs' QOL.

\section{Declaration of Conflicting Interests}

The authors declared no potential conflicts of interest with respect to the research, authorship, and/or publication of this article.

\section{Funding}

The authors disclosed receipt of the following financial support for the research, authorship, and/or publication of this article: This research was partially supported by a grant awarded to the UNC Institute on Aging by the National Institute on Aging (NIA) Pre-Doctoral/PostDoctoral Traineeship, Grant No. 5T32AG000272. The original studies for this current study were supported by grants from the Robert Wood Johnson Foundation; the AARP Andrus Foundation; the Retirement Research Foundation; the National Institute on Aging (P50 AG08012); and the National Institute of Mental Health (RO1MH070629). 


\section{References}

1. Alzheimer's Association. 2012 Alzheimer's disease facts and figures. 2012;8(2):131-168.

2. Hebert LE, Weuve J, Scherr PA, Evans DA. Alzheimer disease in the United States (2010-2050) estimated using the 2010 census. Neurology. 2013;80(19):1778-1783.

3. Phelan EA, Debnam KJ, Anderson LA, Owens SB. A systematic review of intervention studies to prevent hospitalizations of community-dwelling older adults with dementia. Med Care. 2015;53(2):207-213.

4. Whitlatch CJ, Feinberg LF, Tucke SS. Measuring the values and preferences for everyday care of persons with cognitive impairment and their family caregivers. Gerontologist. 2005;45(3):370-380.

5. Whitlatch CJ, Piiparinen R, Feinberg LF. How well do family caregivers know their relatives' care values and preferences? Dementia. 2009;8(2):223-243.

6. Hirschman KB, Joyce CM, James BD, Xie SX, Karlawish JH. Do Alzheimer's disease patients want to participate in a treatment decision, and would their caregivers let them? Gerontologist. 2005;45(3):381-388.

7. Horton-Deutsch S, Twigg P, Evans R. Health care decisionmaking of persons with dementia. Dementia. 2007;6(1): 105-120.

8. Horton AM, Alana S. Validation of the mini-mental state examination. Int J Neurosci. 1990;53(2-4):209-212.

9. Edvardsson D, Innes A. Measuring person-centered care: a critical comparative review of published tools. Gerontologist. 2010; 50(6):834-846.

10. Logsdon RG, Gibbons LE, McCurry SM, Teri L. Assessing quality of life in older adults with cognitive impairment. Psychosom Med. 2002;64(3):510-519.

11. Rubinstein RL, Lawton MP. Depression in Long Term and Residential Care: Advances in Research and Treatment. New York City, NY: Springer Publishing Company; 1997:29-54.

12. Burgener $\mathrm{S}$, Twigg $\mathrm{P}$. Relationships among caregiver factors and quality of life in care recipients with irreversible dementia. Alzheimer Dis Assoc Disord. 2002;16(2):88-102.

13. Logsdon RG, Gibbons LE, McCurry SM, Teri L. Assessing changes in quality of life in Alzheimer's disease: New York, NY: Springer Publications; 2005.

14. Vogel A, Mortensen EL, Hasselbalch SG, Andersen BB, Waldemar G. Patient versus informant reported quality of life in the earliest phases of Alzheimer's disease. Int J Geriatr Psychiatry. 2006; 21(12):1132-1138.

15. Selai CE, Trimble MR, Rossor MN, Harvey RJ. Assessing quality of life in dementia: Preliminary psychometric testing of the Quality of Life Assessment Schedule (QOLAS). Neuropsychol Rehabil. 2001;11(3-4):219-243.

16. Sands LP, Ferreira P, Stewart AL, Brod M, Yaffe K. What explains differences between dementia patients' and their caregivers' ratings of patients' quality of life? Am J Geriatr Psychiatry. 2004;12(3):272-280.

17. Ready RE, Ott BR, Grace J. Patient versus informant perspectives of quality of life in mild cognitive impairment and Alzheimer's disease. Int J Geriatr Psychiatry. 2004;19(3):256-265.
18. Novella J, Jochum C, Jolly D, et al. Agreement between patients' and proxies' reports of quality of life in Alzheimer's disease. Qual Life Res. 2001;10(5):443-452.

19. Logsdon RG, Gibbons LE, McCurry SM, Teri L. Quality of life in Alzheimer's disease: patient and caregiver reports. J Ment Health Aging. 1999;5(1):21-32.

20. Hoe J, Katona C, Orrell M, Livingston G. Quality of life in dementia: care recipient and caregiver perceptions of quality of life in dementia: the LASER-AD study. Int J Geriatr Psychiatry. 2007;22(10):1031-1036.

21. Conde-Sala JL, Garre-Olmo J, Turró-Garriga O, López-Pousa S, Vilalta-Franch J. Factors related to perceived quality of life in patients with Alzheimer's disease: the patient's perception compared with that of caregivers. Int J Geriatr Psychiatry. 2009; 24(6):585-594.

22. Huang HL, Chang MY, Tang JS, Chiu YC, Weng LC. Determinants of the discrepancy in patient- and caregiver-rated quality of life for persons with dementia. J Clin Nurs. 2009;18(22): 3107-3118.

23. Antonucci TC, Israel BA. Veridicality of social support: a comparison of principal and network members' responses. $J$ Consult Clin Psychol. 1986;54(4):432-437.

24. Kim K, Zarit SH, Eggebeen DJ, Birditt KS, Fingerman KL. Discrepancies in reports of support exchanges between aging parents and their middle-aged children. J Gerontol B Psychol Sci Soc Sci. 2011;66(5):527-537.

25. Dilworth-Anderson P, Gibson BE. The cultural influence of values, norms, meanings, and perceptions in understanding dementia in ethnic minorities. Alzheimer Dis Assoc Disord. 2002;16(suppl 2):s56-s63.

26. Covinsky KE, Newcomer R, Fox P, et al. Patient and caregiver characteristics associated with depression in caregivers of patients with dementia. J Gen Intern Med. 2003;18(12):1006-1014.

27. Pinquart M, Sörensen S. Spouses, adult children, and children-inlaw as caregivers of older adults: a meta-analytic comparison. Psychol Aging. 2011;26(1):1-14.

28. Pearlin LI, Menaghan EG, Lieberman MA, Mullan JT. The stress process. J Health Soc Behav. 1981;22(4):337-356.

29. Judge KS, Menne HL, Whitlatch CJ. Stress process model for individuals with dementia. Gerontologist. 2010;50(3):294-302.

30. Pearlin LI, Mullan JT, Semple SJ, Skaff MM. Caregiving and the stress process: an overview of concepts and their measures. Gerontologist. 1990;30(5):583-594.

31. Menne HL, Judge KS, Whitlatch CJ. Predictors of quality of life for individuals with dementia Implications for intervention. Dementia. 2009;8(4):543-560.

32. McLeod JD. The meanings of stress expanding the stress process model. Soc Ment Health. 2012;2(3):172-186.

33. Feinberg LF, Whitlatch J. Decision-making for persons with cognitive impairment and their family caregivers. Am J Alzheimers Dis Other Demen. 2002;17(4):237-244.

34. Katz S, Ford AB, Moskowitz RW, Jackson BA, Jaffe MW. Studies of illness in the aged: the index of ADL: a standardized measure of biological and psychosocial function. JAMA. 1963; 185(12):914-919. 
35. Lawton M, Brody EM. Assessment of older people: selfmaintaining and instrumental activities of daily living. Gerontologist. 1969;9(3):179-86.

36. Kempen G, Suurmeijer TP. The development of a hierarchical polychotomous ADL-IADL scale for noninstitutionalized elders. Gerontologist. 1990;30(4):497-502.

37. Scott WK, Macera CA, Cornman CB, Sharpe PA. Functional health status as a predictor of mortality in men and women over 65. J Clin Epidemiol. 1997;50(3):291-296.

38. Menne HL, Tucke SS, Whitlatch CJ, Feinberg LF. Decision-making involvement scale for individuals with dementia and family caregivers. Am J Alzheimers Dis Other Demen. 2008;23(1):23-29.

39. RadloffLS. The CES-D scale a self-report depression scale for research in the general population. Appl Psychol Meas. 1977;1(3):385-401.

40. Poulshock SW, Deimling GT. Families caring for elders in residence: issues in the measurement of burden. J Gerontol. 1984; 39(2):230-239.

41. Pett MA, Lackey NR, Sullivan JJ. Making Sense of Factor Analysis: The Use of Factor Analysis for Instrument Development in Health Care Research. Thousand Oaks, CA: SAGE Publications; 2003.

42. Horowitz A, Goodman CR, Reinhardt JP. Congruence between disabled elders and their primary caregivers. Gerontologist. 2004; 44(4):532-542.

43. Curran PJ, West SG, Finch JF. The robustness of test statistics to nonnormality and specification error in confirmatory factor analysis. Psychol Meth. 1996;1(1):16-29.
44. Cohen J. Statistical Power Analysis for the Behavioral Sciences. Cambridge, UK: Academic press; 2013.

45. Edwards H, Noller P. Factors influencing caregiver-care receiver communication and its impact on the well-being older care receivers. Health Commun. 1998;10(4):317-341.

46. Whitlatch $\mathrm{C}$. Informal caregivers: communication and decision making. Am J Nurs. 2008;108(9):73-77.

47. Lyons KS, Zarit SH, Sayer AG, Whitlatch CJ. Caregiving as a dyadic process perspectives from caregiver and receiver. $J$ Gerontol B Psychol Sci Soc Sci. 2002;57(3):p195-p204.

48. Woods R. Discovering the person with Alzheimer's disease: cognitive, emotional and behavioural aspects. Aging Ment Health. 2001;5(suppl 1):7-16.

49. Yesavage JA, Brink T, Rose TL, et al. Development and validation of a geriatric depression screening scale: a preliminary report. J Psychiatr Res. 1983;17(1):37-49.

50. Brod M, Stewart AL, Sands L, Walton P. Conceptualization and measurement of quality of life in dementia: the dementia quality of life instrument (DQoL). Gerontologist. 1999;39(1): 25-36.

51. American Geriatrics Society Expert Panel on Person-Centered Care. Person-centered care: a definition and essential elements. $J$ Am Geriatr Soc. 2016;64(1):15-18.

52. Whitlatch CJ, Judge K, Zarit SH, Femia E. Dyadic intervention for family caregivers and care receivers in early-stage dementia. The Gerontologist. 2006;46(5):688-694. 